\title{
Annotationen und ihre infrastrukturellen Prämissen
}

Philipp Hegel [1], Germaine Götzelmann [2], Michael Krewet [1] \& Danah Tonne [2]

[1] Freie Universität Berlin

[2] Karlsruher Institut für Technologie

http://www.sfb-episteme.de/

Keywords: Annotations, Digital Infrastructure, Collaborative Research Centre

\begin{abstract}
Since 2016 the Collaborative Research Centre 980 "Episteme in Motion" includes an information infrastructure project which combines humanities with informatics and establishes a central infrastructure for all participating projects. The article describes this infrastructure with its more local or more distributed aspects briefly in two different perspectives: as 'eye of a needle' and as 'groundwork'. The example chosen is the use of digital annotations and a Web Annotation Protocol server used by different projects of the Collaborative Research Centre.
\end{abstract}

Als Jean-François Lyotard 1979 seinen „Bericht über das Wissen“ vorlegte, geschah dies auch als Beschreibung jener Veränderungen, denen das Wissen im digitalen Zeitalter unterliege:

„Il est raisonnable de penser que la multiplication de machines informationnelles affecte et affectera la circulation de connaissances autant que l'a fait le développement de moyens de circulation des hommes d'abord (transports), des sons et des images ensuite (media). Dans cette transformation générale, la nature du savoir ne reste pas intacte. II ne peut passer dans les nouveaux canaux, et devinir opérationnel, que si la connaissance peut être traduite en quantités d'information. [...] Avec l'hégémonie de l'informatique, c'est une certaine logique qui s'impose, et donc un ensemble de prescriptions portant sur les énoncés acceptés comme ,de savoir"“1

Diese „Vorherrschaft der Informatik“ stellt sich bei Lyotard als ein Flaschenhals dar, durch den das Wissen fließen muss, wenn es in dieser Welt nicht verlorengehen soll. Diskutiert werden sollen hier nicht die Prämissen und Konsequenzen dieser Auffassung. Es sollen vielmehr zwei Sichtweisen mit Blick auf digitale Forschungsinfrastrukturen und anhand eines Beispiels betrachtet werden, die sich eher lose auf Lyotards Stellungnahme beziehen. Zum einen werden digitale Infrastrukturen und Methoden heute oft als Fundament einer Forschung verstanden, die Fragen anders beantwortet und neue Fragen stellt. ${ }^{2}$ Zum anderen bleibt aber doch mit Lyotard zu überlegen, ob und wie sich ein jeweils gegebener Gegenstand oder eine wissenschaftliche Methode in digitalen Verfahren abbilden lässt.

Jean-François LYOTARD, La condition postmoderne. Rapport sur le savoir, Paris 1979, $12 \mathrm{f}$

Wenn im Folgenden von „Infrastrukturen“ gesprochen wird, sind digitale Forschungsinfrastrukturen gemeint. 
Man kann sich fragen, ob diese Objekte und Verfahren durch das Nadelöhr digitaler Infrastrukturen gezogen werden und dabei ,intakt' bleiben können. Diese Punkte betreffen die Frage nach den Bedingungen und Funktionen einer Infrastruktur für die Erzeugung, Tradierung, Wandlung und Nutzung eines bestimmten Wissens und damit auch die Frage nach der Modellierung der Daten und Verfahren.

Digitale Infrastrukturen sollen im Allgemeinen Daten für die und aus der Forschung dauerhaft und zugänglich speichern. ${ }^{3}$ Hinzu kommt die Entwicklung, Einbindung und Pflege von Werkzeugen und Diensten. ${ }^{4}$ Im konkreten Fall umfassen diese beiden Aspekte unter anderem ein Repositorium, einen Server zur Speicherung digitaler Annotationen in einem RDF Triple Store mit SPARQL-Endpoint, ein Werkzeug zur semiautomatischen Layoutanalyse und ein Werkzeug zur digitalen Annotation ihrer Ergebnisse. ${ }^{5}$ Die erforderliche Modellierung der Daten und Annotationen ist auch eine fachliche Entscheidung und kann Auswirkungen auf die fachwissenschaftliche Sicht selbst haben. ${ }^{6}$

Es gibt den Vorschlag, zwischen Forschungsinfrastrukturen lokaler und distribuierter Art zu unterscheiden. Die einen stellten eine „bestimmte Menge von Daten und/oder Werkzeugen zur Verfügung“, die anderen sollen „Standards definieren und Services bereitstellen, welche es erlauben, eine Vernetzung von Daten und Werkzeugen zu ermöglichen und/oder Lösungen für einzelne Fachbereiche oder Problemfelder bereitzustellen“. ${ }^{7}$ Bei diesem Vorschlag wird darauf hingewiesen, dass "die Grenzen hier durchaus fließend verlaufen“. ${ }^{8}$ Eine Infrastruktur innerhalb eines Sonderforschungsbereichs wie die des hier behandelten SFB 980 „Episteme in Bewegung“ kann die Aufgabe haben, Daten aus einzelnen Projekten zu sammeln und Werkzeuge bereitzustellen, aber auch die, die Daten aus Projekten und die dort verwendeten Werkzeuge miteinander zu verbinden. Sie definiert meist keine Standards, kann aber festlegen, welche Standards verwendet werden können und sollten. ${ }^{9}$ Daher scheint es schwierig, sie in dieser Unterscheidung eindeutig zu verorten.

Will man nun die metaphorische Rede vom ,Nadelöhr' oder vom ,Fundament‘ präzisieren, kann dies mit Blick auf die Bedingungen und Funktionen der einzelnen technischen Komponenten geschehen.

3 Heike Neuroth / Andrea Rapp, Nachhaltigkeit von digitalen Infrastrukturen, in: Bibliothek - Forschung und Praxis 40/2 (2016), 264-270, hier 268, nennen dies „datentechnische Nachhaltigkeit“. Sie beschäftige sich „vordergründig mit dem Nachweis, der Nachnutzung und der persistenten Speicherung von digitalen wissenschaftlichen Sammlungen und Forschungsdaten der Geistes- und Kulturwissenschaften." Schon in diesem Punkt werden auch die gesellschaftlichen und kulturellen Rahmenbedingungen einer Infrastruktur deutlich. Bei der Sammlung des Aristotelismus-Zentrums Berlin, die für das später behandelte Beispiel relevant ist, betrifft dies zum Beispiel die unterschiedlichen Rechte an Mikrofilmen und Digitalisaten von Bibliotheken aus aller Welt.

4 Ebd., 268, bezeichnen Neuroth und Rapp dies als „technische Nachhaltigkeit“ und meinen damit nicht nur die „Stabilisierung der bisherigen Dienste [...], sondern [...] den kompletten Service-Lifecycle." Dies lässt sich sowohl auf die „datentechnische" Komponente als auch auf weitere Werkzeuge und Dienste beziehen.

5 Etwas ausführlicher dargestellt in Danah TonNE u.a., Ein Web Annotation Protocol Server zur Untersuchung vormoderner Wissensbestände, in: Patrick Sahle, Hg., Digital Humanities im deutschsprachigen Raum 2019: Multimedial und multimodal, Frankfurt am Main 2019, 285-288, online unter: https://zenodo.org/record/2596095 (25.03.2019).

6 Die Bedeutung der Modellierung auch für digitale Forschung ist mehrfach hervorgehoben worden. Vgl. zum Beispiel Willard McCARTY, Modeling, A Study in Words and Meanings, in: Susan Schreibman / Ray Siemens / John Unsworth, Hg., A Companion to Digital Humanities, Oxford 2004, online unter: http://www.digitalhumanities.org/companion (25.03.2019), der zu diesem Verhältnis schreibt: „modeling of something readily turns into modeling for better or more detailed knowledge of it; similarly, the knowledge gained from realizing a model for something feeds or can feed into an improved version." Hervorhebungen im Original.

7 So Gerhard HEYER / Thomas ECKART / Dirk GoldHAHN, Was sind IT-basierte Forschungsinfrastrukturen für die Geistes- und Sozialwissenschaften und wie können sie genutzt werden?, in: Information. Wissenschaft \& Praxis 66/5-6 (2015), 295303, hier 296.

8 Ebd., 296.

9 Ebd., 296, scheinen Heyer, Eckart und Goldhahn diesen schwächeren Sinn von ,definieren' zumindest unter anderem zu meinen, wenn sie erläutern, dass die Werkzeuge von Forschungsinfrastrukturen „von den definierten Standards und bereitgestellten Services im Sinne einer Integration und Interoperabilität Gebrauch machen“. 
Funktionen können oft variabel, aber nicht beliebig realisiert werden. Hat man ein digitales Werkzeug ausgewählt, sind ,Input', Funktionalität und ,Output' mehr oder weniger strikt festgelegt. ${ }^{10}$ In einer Infrastruktur stehen technische Komponenten zudem nicht für sich allein, sondern reagieren auf die Bedingungen und Möglichkeiten anderer Komponenten und bedingen und ermöglichen zugleich deren Verwendung. ${ }^{11}$ Diese Bedingungen und Funktionen der Komponenten sind auch die Grundlage für ihre Nutzung in verschiedenen Forschungsvorhaben und damit für die Möglichkeit ,distribuierter Infrastrukturen.

Digitale Annotationen bieten sich für ein solche Distribution in einem Verbund an. Erstens ist bei innen die mediale Entkopplung von Anmerkung und annotiertem Objekt zu bemerken. ${ }^{12}$ Infrastrukturelle Stabilität, ${ }^{13}$ wie sie ein Repositorium leisten kann, sowie Möglichkeiten der Referenzierung und Verknüpfung vorhandener Daten ${ }^{14}$ vorausgesetzt, können Annotationen kollaborativ am digitalen Objekt vorgenommen werden. In einem Vorhaben zu etwa 150 griechischsprachigen Handschriften der Überlieferung von De interpretatione eröffnen digitale Annotationen zu Scholien, Diagrammen und Glossen beispielsweise die Möglichkeit, diese Hinzufügungen zu transkribieren und ihre Herkunft zu kennzeichnen, um Wanderungen von einer zur nächsten Handschrift, von einem zu einem anderen Ort nachvollziehbar werden zu lassen. Stellen werden dabei mit projektspezifischen Begriffslisten oder kontrollierten Vokabularen klassifiziert und auf unterschiedlichen Ebenen erschlossen. Die digitalen Annotationen konnten systematisch ausgewertet werden und so in Kombination mit den Handschriftenbeschreibungen exemplarisch einen Textzeugen in einem überlieferungsgeschichtlichen Zweig positionieren, in den man sie, ginge man nur vom aristotelischen Text aus, kaum einordnen würde.

Digitale Annotationen, wie sie das Web Annotation Data Model des W3C-Konsortiums definiert, verhalten sich zweitens relativ neutral zu Medium und Inhalt sowohl der Annotation („body“) als auch des annotierten Gegenstands („target“). Dies erleichtert die Übertragung der Verfahren von einem Projekt wie dem beschriebenen auf ein anderes Forschungsvorhaben etwa zu frühneuzeitlichen Sprachlehrwerken. Die technischen Komponenten werden in diesem Sinne ,distribuiert' eingesetzt, erfordern aber eine spezifische Datenmodellierung und -auswertung, um diese Komponenten ,lokal' in einem konkreten Forschungsprozess und damit fachwissenschaftlich zu verankern.

10 Der ursprünglich psychologisch geprägte Begriff der ,Affordanz' wird in jüngerer Zeit auch zur Beschreibung des Verhältnisses zwischen Akteuren und Artefakten und auch auf digitale Infrastrukturen angewandt. Ulla TscHIDA, Auf der Suche nach dem Artefakt. Zur materiellen Praxis von Infrastruktur-Entwicklung, in: Friederike Elias u.a., Hg., Praxeologie. Beiträge zur interdisziplinären Reichweite praxistheoretischer Ansätze in den Geistes- und Sozialwissenschaften, Berlin 2014, 219-242, hier 221, übernimmt die entsprechende Explikation Andreas Reckwitz und erläutert dies in Hinblick auf ihren Gegenstand: „Die immanenten Strukturen eines technischen Artefakts legen eine Verwendungsweise nahe, determinieren sie aber nicht. Gleichzeitig sind die Handlungsmodi nicht beliebig - die Objekteigenschaften ermöglichen bestimmte Umgangsweisen, andere sind ausgeschlossen." Im Sinne einer wissensgeschichtlichen Wendung könnte ergänzt werden, dass das Wissen der Akteure um diese Bedingungen und Möglichkeiten ausschlaggebend für ihren Umgang mit Artefakten und Infrastrukturen ist.

11 Ebd., 233, erwägt Tschida hierfür ebenfalls die Beschreibung über Affordanzen: „Ohne den Begriff der Affordances überstrapazieren zu wollen, stellt sich die Frage, ob technische Strukturen nicht auch variable, nicht-arbiträre Angebotsstrukturen für andere technische Strukturen aufweisen“.

12 Vgl. Harald LoRDick u.a., Digitale Annotationen in der geisteswissenschaftlichen Praxis, in: Bibliothek - Forschung und Praxis 40/2 (2016), 186-199, hier 187: „Nicht zuletzt führt die physische Entkoppelbarkeit der Annotation vom annotierten Objekt zu einer Veränderung des Objekts Annotation selbst.“

13 Vgl. ebd., 196: „Die Verfügbarkeit von Daten spielt eine entscheidende Rolle - zum einem im Hinblick auf annotierbare digitale Gegenstände, zum anderen auf Daten, die als Annotationsinhalte oder Anreicherungen genutzt werden können, z.B. Normdaten."

14 Vgl. ebd., 195: „Für das webbasierte semantische Annotieren ist charakteristisch, [...] Metadaten nicht nur mit eindeutigen Identifikatoren zu versehen, um ihre Interoperabilität und eindeutige Referenzierbarkeit zu gewährleisten, sondern darüber hinaus Verknüpfungen zwischen Ressourcen herzustellen und diese zu typisieren.“ 


\section{Literaturverzeichnis}

Gerhard HEYER / Thomas ECKART / Dirk GoLDHAHN, Was sind IT-basierte Forschungsinfrastrukturen für die Geistes- und Sozialwissenschaften und wie können sie genutzt werden?, in: Information. Wissenschaft \& Praxis 66/5-6 (2015), 295-303.

Harald LORDICK u.a., Digitale Annotationen in der geisteswissenschaftlichen Praxis, in: Bibliothek - Forschung und Praxis 40/2 (2016), 186-199.

Jean-François LYOTARD, La condition postmoderne. Rapport sur le savoir, Paris 1979.

Willard McCARTY, Modeling, A Study in Words and Meanings, in: Susan Schreibman / Ray Siemens / John Unsworth, Hg., A Companion to Digital Humanities, Oxford 2004, online unter: http://www.digitalhumanities.org/ companion (25.03.2019).

Heike NEUROTH / Andrea RaPP, Nachhaltigkeit von digitalen Infrastrukturen, in: Bibliothek - Forschung und Praxis 40/2 (2016), 264-270.

Danah TonNe u.a., Ein Web Annotation Protocol Server zur Untersuchung vormoderner Wissensbestände, in: Patrick Sahle, Hg., Digital Humanities im deutschsprachigen Raum 2019: Multimedial und multimodal, Frankfurt am Main 2019, 285-288, online unter: https://zenodo.org/record/2596095 (25.03.2019).

Ulla TschiDA, Auf der Suche nach dem Artefakt. Zur materiellen Praxis von Infrastruktur-Entwicklung, in: Friederike Elias u.a., Hg., Praxeologie. Beiträge zur interdisziplinären Reichweite praxistheoretischer Ansätze in den Geistes- und Sozialwissenschaften, Berlin 2014, 219-242. 